\title{
Tetrandrine mediates renal function and redox homeostasis in a streptozotocin-induced diabetic nephropathy rat model through Nrf2/HO-1 reactivation
}

\author{
Luyu Su ${ }^{1}$, Ping $\mathrm{Cao}^{2}$, Haiyan Wang ${ }^{3}$ \\ ${ }^{1}$ Department of Endocrinology Division, Shaanxi Traditional Chinese Medicine University Affiliated Hospital, Xianyang, China; ${ }^{2}$ Department \\ of ophthalmology, Shaanxi Traditional Chinese Medicine University Affiliated Hospital, Xianyang, China; ${ }^{3}$ Department of Endocrinology, Xi'an \\ International Medical Center Hospital, Xi'an, China \\ Contributions: (I) Conception and design: H Wang, L Su; (II) Administrative support: H Wang; (III) Provision of study materials or patients: H Wang, \\ P Cao; (IV) Collection and assembly of data: L Su, P Cao; (V) Data analysis and interpretation: L Su, P Cao; (VI) Manuscript writing: All authors; (VII) \\ Final approval of manuscript: All authors. \\ Correspondence to: Haiyan Wang. Department of Endocrinology, Xi'an International Medical Center Hospital, Fifty Meters East of Xitai First Class \\ Highway, Xi'an, China. Email: sunshade98121@163.com.
}

Background: Diabetic nephropathy $(\mathrm{DN})$ is the leading cause of morbidity and mortality in diabetic patients. Tetrandrine (Tet), a bisbenzylisoquinoline alkaloid isolated from the roots of Stephania tetrandra, possesses anti-oxidative, anti-hypertensive, anti-inflammatory capacities. In this study, the maintenance role of Tet in DN was evaluated in streptozotocin (STZ)-induced diabetic rats.

Methods: In vitro study, rats were divided into five groups $(\mathrm{n}=10)$ : the control group, the DN model group, the Tet-treatment group $(5,15,30 \mathrm{mg} / \mathrm{kg})$. DN damage was assessed by levels of blood glucose, serum creatinine (CRE), proteinuria, and urea nitrogen. ELISA assay was used to detecte tumor necrosis factor- $\alpha$ (TNF- $\alpha$ ), inducible nitric oxide synthase (iNOS), interleukin-6 (IL-6) and IL-10 levels. Kits were used to detecte contents of malondialdehyde (MDA), lactate dehydrogenase (LDH) and superoxide dismutase (SOD). Dichlorofluorescein (DCF) staining was used to detecte reactive oxygen species (ROS). HE staining assessed pathological damage. TUNEL staining assessed tissue apoptosis. Western Blot (WB) was used to detecte levels of Ki67, Survivin, Bax, Bcl-2, caspase-3, -9, c-Myc, nuclear factor erythroid-derived 2-related factor 2 (Nrf2), p-Nrf2, and heme oxygenase-1 (HO-1).

Results: Compared with the control group, STZ-induced significantly inhibited proliferation proteins' level, activated oxidative stress, aggravated tissue inflammation and promoted tissue apoptosis. STZ-induced further aggravated DN damage. Of note, these anomalies were restored by Tet pretreatment. Additionally, Tet upgraded the expression of p-Nrf2 and HO-1.

Conclusions: These results indicated that Tet could significantly restrain diabetic process and renal damage. Tet is a potential therapeutic agent in DN treatment via the reactivation of the Nrf2/HO- 1 .

Keywords: Diabetic nephropathy (DN); tetrandrine (Tet); oxidative stress; renal function; nuclear factor erythroid-derived 2-related factor 2/heme oxygenase-1 (Nrf2/HO-1)

Submitted Jun 20, 2020. Accepted for publication Aug 05, 2020.

doi: $10.21037 / \mathrm{atm}-20-5548$

View this article at: http://dx.doi.org/10.21037/atm-20-5548

(C) Annals of Translational Medicine. All rights reserved. 


\section{Introduction}

Diabetic nephropathy (DN) is the main reason for morbidity and mortality in diabetic patients (1). The pathogenesis of DN, which involves many pathways, is multifactorial and complex. Hyperglycemia is the primary driver of diabetic glomerular progressive destruction (2). Continued elevated blood glucose can lead to changes in glomerular mechanical tension and hemodynamics, leading to changes in downstream transcription factors and gene expression (3). It is well-known that DN alters not only the structure but also the function of the kidney. These alterations are related to upregulated levels of reactive oxygen species, proinflammatory molecules, and matrix proteins.

Further, the inflammation curtails gap junctional communication and upsurges hemichannel activity leading to increased membrane permeability and changing tissue homeostasis. The variation stimulates several oxidative stress pathways and releases many growth factors and cytokines (4). Hyperglycemia-mediated oxidative stress and inflammatory response are to be recognized as the critical components in DN. Therefore, treatment of inflammation and oxidative stress can effectively support normal renal function and delay $\mathrm{DN}$ progression.

Tetrandrine (Tet), a bisbenzylisoquinoline alkaloid, is isolated from the roots of Stephania tetrandra (5). Extensive researches have been reported that Tet can be broadly used for patients with arthritis (6), hypertension (7), inflammation (8). Tet also exerts an anti-oxidative role in a rat model of diabetes mellitus (9). Moreover, Tet treatment decreases proinflammatory cytokines in PMA plus A23187induced HMC-1 cells (10). Bhagya et al. showed that Tet is a potential target for breast and pancreatic cancers via mediating the reaction of oxygen species- caspase activation pathway (11). However, little information is available about the effect of Tet on DN.

Nuclear factor erythroid-derived 2-related factor 2 (Nrf2) plays a vital role in the system of oxidative stress defense. In normal cells, Nrf2 is isolated in the cytoplasm by physical interaction with the inhibitor Kelch like epichlorohydrin associated protein 1 (Keap1) (12). Responding to various cell damage, Nrf2 escaped the inhibition of Keap1 and aggregated in the nucleus to activate ARE-driven genes expression, including heme oxygenase-1 (HO-1), glutathione S-transferase (GST) and $\mathrm{NAD}(\mathrm{P}) \mathrm{H}$ : quinone oxidoreductase 1 (NQO1) (13). Some studies reported that $\mathrm{Nrf2}$ is associated with renal disease progression (14). The apparent damage to Nrf2 activation can aggravate the progress of inflammation, oxidative stress, and kidney tissue damage (15); thence, activation of Nrf2 therapeutic was regarded as a prevention strategy for DN progression (16). HO-1 is a critical antioxidant enzyme regulated by Nrf2. HO-1 regulates ROS levels in cells to respond to different stimuli. HO-1 regulates not an only cellular antioxidant defense but also has an antiinflammatory effect (17). Since current treatments for DN do not prevent renal damage, we presuppose an alternative treatment by extract from Chinese herbal medicine with antioxidant, anti-inflammatory, and hypoglycemic effects.

However, the effect of Tet on DN and the possible association between Tet and Nrf2/HO-1 in the development of DN has not been clarified. Streptozotocin (STZ)-induced diabetes was proved to be an excellent model for DN early changes studying (18). Thus, in this study, the DN experimental model of rat induced by STZ was set up to reveal the potential role of Tet for mediating renal function and redox homeostasis. Furthermore, the underlying mechanisms of Tet on STZ-induced DN was investigated.

We present the following article in accordance with the ARRIVE reporting checklist (available at http://dx.doi. org/10.21037/atm-20-5548).

\section{Methods}

\section{Materials and methods}

\section{Reagents}

Tet (purity $\geq 98 \%$ ) was bought from Yaji Biotechnology Co., Ltd. (Shanghai, China). The kits for hemoglobinuria, malondialdehyde (MDA), lactate dehydrogenase (LDH), superoxide dismutase (SOD), creatinine (CRE) and blood urea nitrogen (BUN) were purchased from Shanghai Qiaoyu Biotechnology Co., Ltd. (Shanghai, China). The IL-10, IL-6, inducible nitric oxide synthase (iNOS), and tumor necrosis factor- $\alpha(\mathrm{TNF}-\alpha)$ kits were bought from R\&D Systems Co., Ltd. (Minneapolis, MN, USA). STZ was bought from Sigma (St. Louis, MO, USA).

\section{Animals models}

A total of 50 Wistar rats (6-8 weeks old; $100-200 \mathrm{~g}$; sex ratio, 1:1) were purchased from Shaanxi Provincial Center for Disease Control and Prevention (Xi'an, Shaanxi, China). Animals were raised in a controlled environment (12 hours light/dark cycle; $24 \pm 2{ }^{\circ} \mathrm{C} ; 55-60 \%$ humidity). All rats had 
free access to water and food.

The groups were divided randomly $(\mathrm{n}=10)$ : the control group, the DN model group, the Tet-treatment group (the high-dose Tet group: $30 \mathrm{mg} / \mathrm{kg}$; the medium-dose Tet group: $15 \mathrm{mg} / \mathrm{kg}$; the low-dose Tet group: $5 \mathrm{mg} / \mathrm{kg}$ ). At the end of the adaptation period, the control group rats were continued to be fed with standard feed, DN model group and Tet treatment group were injected with lowdose STZ $(35 \mathrm{mg} / \mathrm{kg})$ diluted with citrate buffer $(0.1 \mathrm{~mol} / \mathrm{L}$ sodium citrate and $0.1 \mathrm{~mol} / \mathrm{L}$ citric acid, $\mathrm{pH} 4.5$ ) (19). Particularly, the DN model group and the Tet-treatment group were given a high-fat diet to stimulate diabetes until the experiment over. Diabetes was determined by estimating blood glucose levels $(>16.7 \mathrm{mmol} / \mathrm{L})$ on the $3 \mathrm{rd}$ day after STZ administration. The second day after the establishment of the diabetes model, the rats were treated with Tet at different concentrations daily for eight weeks. The control group and the DN model group were given an equal volume of distilled water. The dose of Tet used in animal models was selected following the previous report and was improved (20). ML-385, the specific inhibitor of $\mathrm{Nrf2}$, was used in our study to make the results more credible. The study was approved by the ethical committee of Shaanxi Traditional Chinese Medicine University Affiliated Hospital (Xianyang, China) (No. SXZY-1907). All animal experiments were conducted according to the ethical guidelines of Shaanxi Traditional Chinese Medicine University Affiliated Hospital (Xianyang, China).

\section{Biochemical assays}

Weekly, the levels of blood glucose from the tail vein were detected by venipuncture with a glucose meter (Bayer HealthCare LLC, NY, USA).

Blood samples were collected and centrifuged at 3,000 rpm for $10 \mathrm{~min}$ to separate plasma. Serum CRE, proteinuria, and urea nitrogen were detected with diagnostic kits.

\section{Histopathology}

Kidney tissues were treated with hematoxylin and eosin $(H \& E)$ staining. Kidney tissues were fixed in $4 \%$ paraformaldehyde for 24 hours and embedded in paraffin. The tissue was sectioned about $4 \mu \mathrm{m}$. The slices were stained with hematoxylin, eosin, and toluidine blue and then were used to observe the pathological changes of the tissues under light microscopy.

\section{ELISA detected the levels of inflammatory factors $I L-10$, IL-6, iNOS, TNF- $\alpha$ in serum}

The pretreatment medium of the kidney tissues were stored at $-80{ }^{\circ} \mathrm{C}$ until measurements. IL-10, IL-6, iNOS, and TNF- $\alpha$ levels in kidney tissues were detected with ELISA kits.

\section{The kit detected oxidative stress markers}

The levels of malondialdehyde (MDA) were detected using the thiobarbituric acid (TBA) method, according to the instructions of the manufacture. The OD value measured at 530 and $600 \mathrm{~nm}$, and the content was conversed according standard curve data.

With SOD assay Kit, SOD activity was assessed by quantifying formazan dye levels. Formazan dye is one kind of substance that is eliminated by SOD and produced by the reaction between xanthine oxidase $(\mathrm{XO})$ and wst-8. Upon $30 \mathrm{~min}$ of incubation at $37^{\circ} \mathrm{C}$, OD values were measured at $450 \mathrm{~nm}$. The activity of SOD enzyme was calculated according to the reduction in formazan dye content.

Kidney injury was evaluated by measure the lactate dehydrogenase (LDH) activity using the cytotoxicity detection kit according to the manufacturer's instructions. $O D$ values were measured at 490 and $600 \mathrm{~nm}$, and the content was conversed according standard curve data.

\section{ROS generation}

ROS level was detected following previous literature (21). Firstly, the kidney tissue was incubated in Hank's balanced salt solution with $5 \mathrm{mM} \mathrm{H} 2 \mathrm{DCFDA}$ for $1 \mathrm{~h}$. Upon oxidation, the nonfluorescent H2DCFDA was converted to the highly fluorescent 2 ', 7 '-dichlorofluorescein (DCF). The fluorescence of DCF was measured at an excitation wavelength of $488 \mathrm{~nm}$ and an emission wavelength of 515-540 nm using an Olympus confocal microscope. Cells images were also obtained by digital interference contrast (DIC). The mean fluorescence intensity of each cell and the number of ROS-positive cells per field were analyzed using Image J software.

\section{Terminal-deoxynucleoitidyl transferase mediated nick end labeling (TUNEL)}

After being dewaxed with xylene, the paraffin sections were hydrated with gradient ethyl alcohol. Then, the sections 

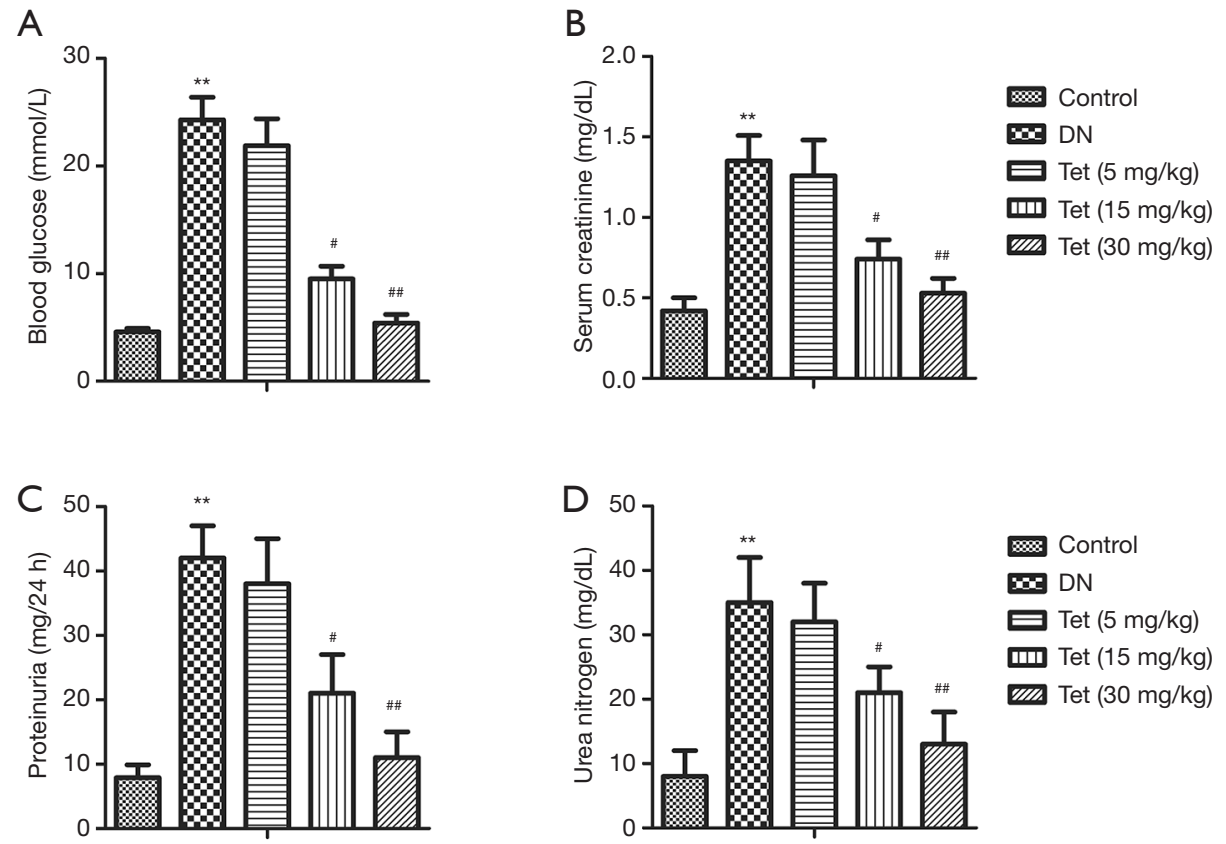

Figure 1 Tet treatment relieves kidney injuries in STZ-induced DN rats. Blood glucose was detected by venipuncture with a glucose meter. (A) The content of blood glucose. The renal function was evaluated by the level of serum creatinine, proteinuria, and urea nitrogen. (B,C,D) The levels of serum creatinine, proteinuria, and urea nitrogen. (**, $\mathrm{P}<0.01$ versus the control group; ${ }^{*} \mathrm{P}<0.05$, \#\#, $\mathrm{P}<0.01$ versus the $\mathrm{DN}$ model group). STZ, streptozotocin; DN, diabetic nephropathy; Tet, tetrandrine.

were cleaned with $3 \%$ methanol peroxide and $20 \mu \mathrm{M}$ proteinase K. Next, the sections were cleaned with PBS and incubated with a mixture of fluorescein reagent at $37^{\circ} \mathrm{C}$ for $60 \mathrm{~min}$. The sections were then stained with DAPI to show the nucleus. Olympus fluorescence observed Apoptosis microscopy (Shinjuku, Tokyo, Japan).

\section{Western blot (WB) analysis}

Proteins were dissolved in RIPA and centrifuged at 12,000 g at $4{ }^{\circ} \mathrm{C}$ for $10 \mathrm{~min}$. Electrophoresis separated protein samples and then transferred to the PVDF membrane. After being sealed with $5 \%$ skimmed milk powder in Tris buffer saline (TBS) with $0.1 \%$ Tween-20 (TBST) for one hour at room temperature, the closed membrane was incubated overnight with primary antibody at $4{ }^{\circ} \mathrm{C}$. The primary antibodies as following: Ki67 (ab15580, 1:500), survivin (ab134170, 1:500), Bax (ab32503, 1:1,000), Bcl-2 (ab32124, 1:1,000),Caspase-3 (ab13847, 1:500), Caspase-9 (ab184786, 1:1,000), c-Myc (ab32072, 1:1,000), p-Nrf2 (ab180844, 1:500), Nrf2 (ab137550, 1:500), HO-1 (ab189491, 1:2,000). The PVDF membranes were cleaned with TBST. Then, they were incubated with horseradish peroxidase (HRP)- bound secondary antibody for 1 hour at room temperature. Finally, they were detected and quantified with the image analysis program.

\section{Statistical analysis}

Analyses were performed using SPSS 22.0 (Chicago, IL, USA). ANOVA or Student t-tests were performed to assess statistical significance, as shown. Data were presented as the mean \pm standard deviation (SD).

\section{Results}

\section{Tet treatment relieves kidney injuries in STZ-induced DN rats}

To evaluated Tet's effect on renal injuries and histopathological changes in STZ stimulated diabetic rats, blood glucose, serum CRE, proteinuria, and urea nitrogen were determined. From Figure 1A, compared with the control group, the blood glucose levels in the DN model group were markedly elevated $(\mathrm{P}<0.01)$. The levels of serum $\mathrm{CRE}$, proteinuria, and urea nitrogen in the DN model group significantly 
A

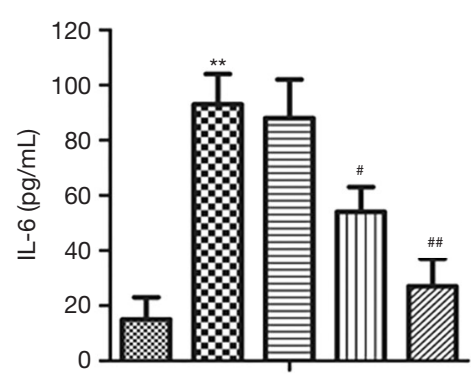

C

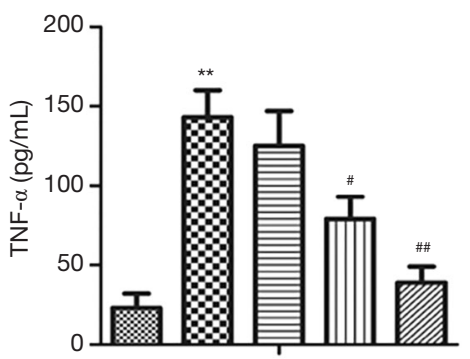

B

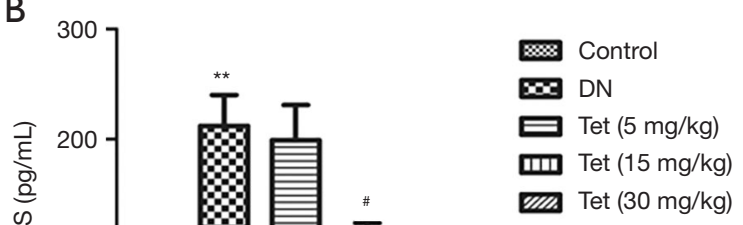

E
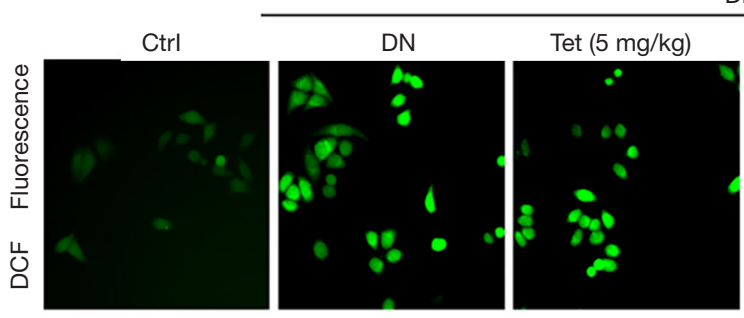

DN
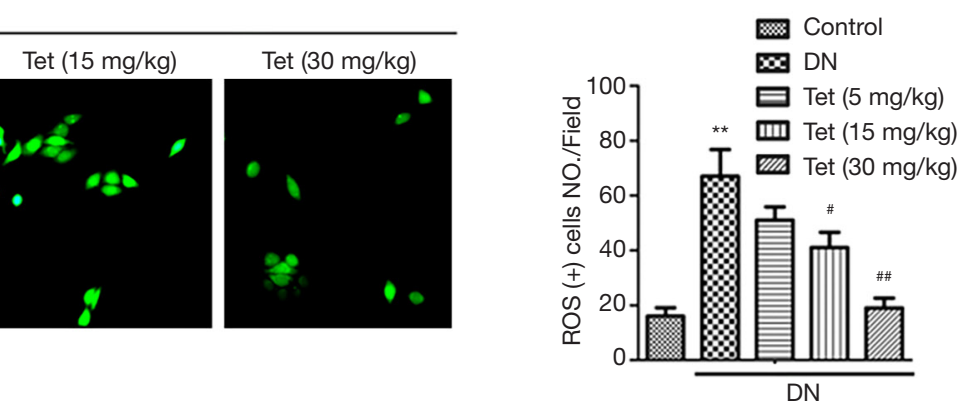

Figure 2 Tet mitigates renal inflammation in STZ-induced DN rats. The productions of IL-6 (A), iNOS (B), TNF- $\alpha$ (C) and IL-10 (D) in kidneys tissue were detected by ELISA kits. (E) The generation of ROS $(\times 200)$. $\left({ }^{* *}, \mathrm{P}<0.01\right.$ versus the control group; ${ }^{\#}, \mathrm{P}<0.05,{ }^{\#}, \mathrm{P}<0.01$ versus the DN model group). STZ, streptozotocin; DN, diabetic nephropathy; Tet, tetrandrine; IL-6, interleukin-6; iNOS, inducible nitric oxide synthase; TNF- $\alpha$, tumor necrosis factor- $\alpha$; IL-10, interleukin-10.

increased compared with the control group $(\mathrm{P}<0.01)$. Tet treatment significantly curtail levels of blood glucose, serum CRE, proteinuria, and urea nitrogen throughout the entire experimental period (Figure 1B,C,D). The results showed that Tet treatment could relieve pathological histological changes and kidney injuries in the STZ-induced DN model.

\section{Tet mitigates renal inflammation in STZ-induced DN rats}

ELISA measured the levels of inflammatory factors in serum. Compared with the control group, the levels of TNF- $\alpha$, IL-6, and iNOS in the DN model group were significantly elevated $(\mathrm{P}<0.01)$. In turn, treatment with Tet significantly reduced levels of inflammatory factors IL-6, TNF- $\alpha$, and iNOS in the serum compared with the DN model group (Figure $2 A, B, C ; \mathrm{P}<0.01$ ). The change of IL-10 level in the DN model group is insignificant to those in the control group. While Tet treatment significantly elevated the level of IL-10 in a concentration-dependent manner (Figure 2D). The results showed that Tet treatment could mitigate renal inflammation in STZ-induced diabetic rats.

\section{Tet improves oxidative stress in STZ-induced DN rats}

Four typical oxidative stress markers were determined to evaluate renal tissue damage. The oxidative stress state is apparent in DN. As shown in Table 1, compared with the 
Table 1 Tet improves oxidative stress in STZ-induced DN rats

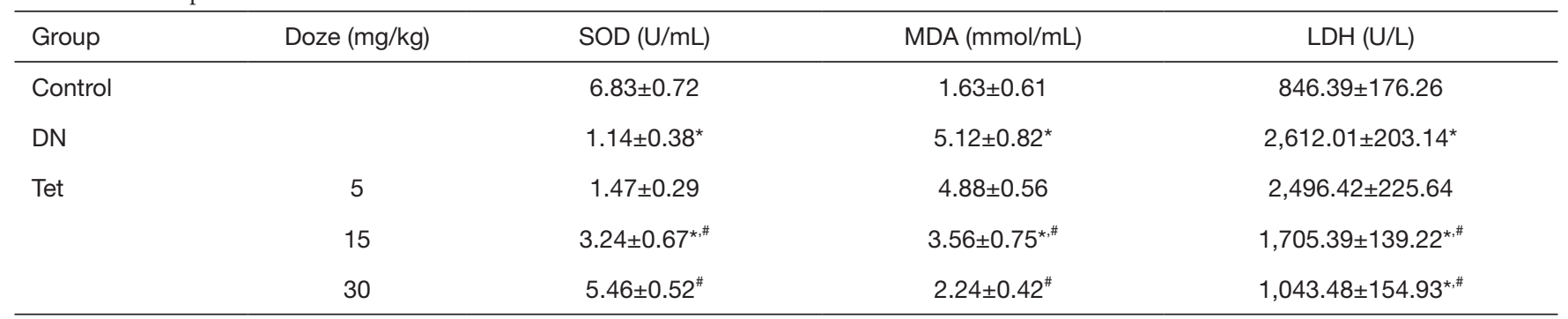

*, $\mathrm{P}<0.05 ;{ }^{* *}, \mathrm{P}<0.01$ versus the control group;, $\mathrm{P}<0.05 ;{ }^{\# \#}, \mathrm{P}<0.01$ versus the DN model group. STZ, streptozotocin; DN, diabetic nephropathy; SOD, superoxide dismutase; MDA, malondialdehyde; LDH, lactate dehydrogenase; Tet, tetrandrine.

control group, levels of MDA, and the leakage of LDH in the DN model group were enhanced obviously $(\mathrm{P}<0.01$, $\mathrm{P}<0.01)$. Furthermore, compared with the control group, the content of SOD in the DN model group was decreased $(\mathrm{P}<0.01)$. From Figure $2 E$, compared with the control group, the generation of ROS in the DN model group was increased $(\mathrm{P}<0.01)$. Interestingly, these oxidative stress phenomenons were improved under Tet treatment. These results showed that Tet could improve oxidative stress damage, which generates in STZ-induced diabetic rats.

\section{Tet relieves pathological histological and tissue apoptosis in the kidneys via regulating the expression of Ki67 and survivin}

Morphological changes and apoptosis were respectively detected by H\&E staining and TUNEL (Figure $3 A$ ). As expected, the kidneys of the DN model group showed glomerular hypertrophy and mesangial matrix dilation. Fortunately, Tet treatment reversed these adverse phenomena. The percent of apoptotic cells was significantly exhibited in the DN model group compared with the control group, as shown in Figure 3B. The levels of Ki67 and survivin were also determined. In the $\mathrm{DN}$ model group, Ki67 and survivin protein expression were decreased significantly (Figure 3C,D,E). After treated with different concentrations of Tet, the expression of Ki67 and survivin were elevated evidently (Figure $3 C, D, E$ ). Our findings suggested that Tet relieves pathological histological and tissue apoptosis changes in the kidneys via regulating the expression of Ki67 and survivin.

\section{Tet relieved mitochondrial damage in the kidneys}

Subsequently, the expression of mitochondrial damage marker molecules Bax/Bcl-2, caspase-3, -9, and c-Myc were determined. WB analysis revealed that in the DN model group, Bax expression was increased significantly with the decreasing expression of Bcl-2 (Figure 4A). Fortunately, Tet treatment reversed these adverse phenomena. The ratio of Bax/Bcl-2 was curtailed significantly after treated with Tet (Figure 4B). In the DN model group, the expression of caspase-3, -9 were increased obviously (Figure 4B). Tet treatment relieved this damage well.

The expression of c-Myc curtailed obviously in the DN model group and elevated significantly in the Tet-treatment group in a dose-dependent manner.

In conclusion, Tet has the protective effect of mitigating mitochondrial damage in the kidneys.

\section{Tet activated Nvf2/HO-1 pathway in DN rat model}

We detected Nrf2, p-Nrf2, and HO-1expression to verify that Nrf2 and HO-1 take part in Tet mediated protection against STZ-induced inflammation and oxidative stress. From Figure $5 A, B$, in the $\mathrm{DN}$ model group, the relative protein level of $\mathrm{p}-\mathrm{Nrf2} / \mathrm{Nrf} 2$ and HO-1 in tissue and serum were downregulated markedly compared with the control group $(\mathrm{P}<0.05, \mathrm{P}<0.05)$. Conversely, Tet treatment improved these unusual phenomena. The results showed that Tet activated Nrf2/HO-1 pathway in DN rat model.

\section{Tet mediated STZ-induced DN in rat model via Nrf2/ HO-1 patbway}

To determine whether Tet mediates STZ induced $\mathrm{DN}$ via Nrf2/HO-1 pathway, we added ML-385, the inhibition of Nrf2. From Figure 6A, ML-385 inhibited the activation of Nrf2 by Tet. Correspondingly, ML-385 enhanced the production of blood glucose and urea nitrogen 
A

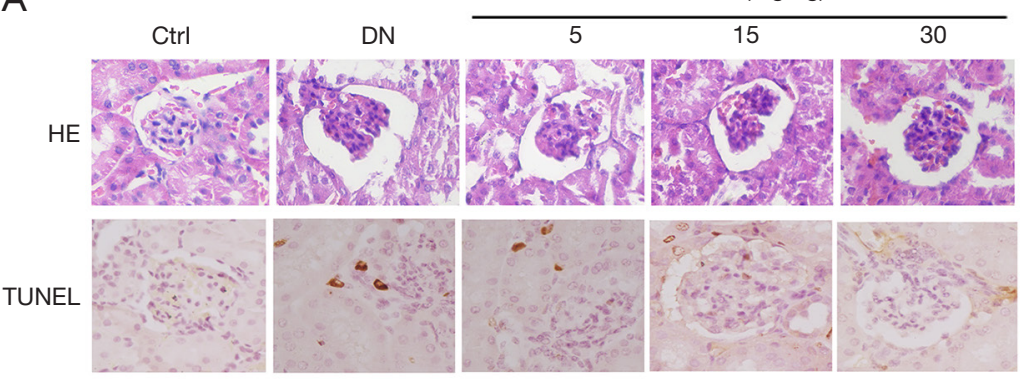

C

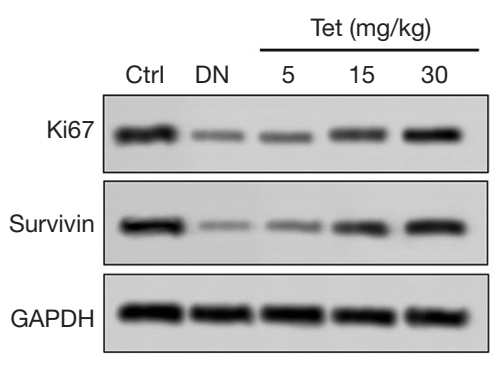

D

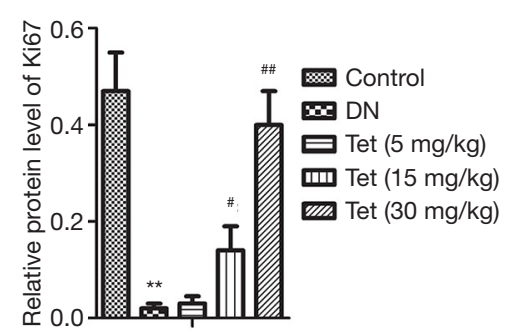

B

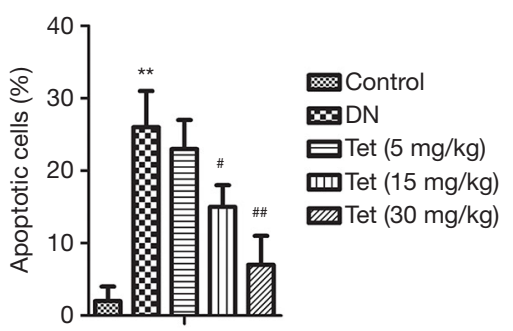

$\mathrm{E}$

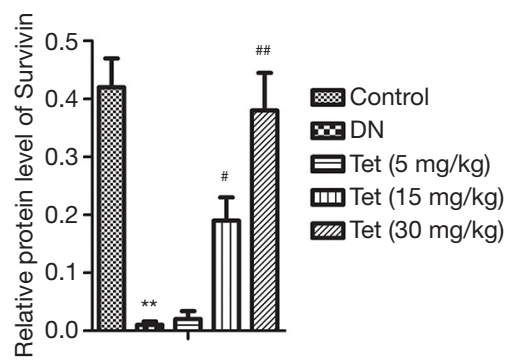

Figure 3 Tet relieves pathological histological and tissue apoptosis in the kidneys via regulating the expression of Ki67 and survivin. Pathological damage was detected by HE, and apoptosis was detected by TUNEL. (A) Representative pictures of HE $(\times 200)$ and TUNEL (×200). (B) Percentage of apoptosis cells. Relative expression of Ki67 and survivin on protein levels were detected by Western blot. (C) Representative pictures of Western blot. (D,E) The relative protein level of Ki67 and survivin. ( ${ }^{* *}, \mathrm{P}<0.01$ versus the control group; \#, $\mathrm{P}<0.05 ;{ }^{\#}, \mathrm{P}<0.01$ versus the $\mathrm{DN}$ model group). DN, diabetic nephropathy; Tet, tetrandrine; TUNEL, terminal-deoxynucleoitidyl transferase mediated nick end labeling.
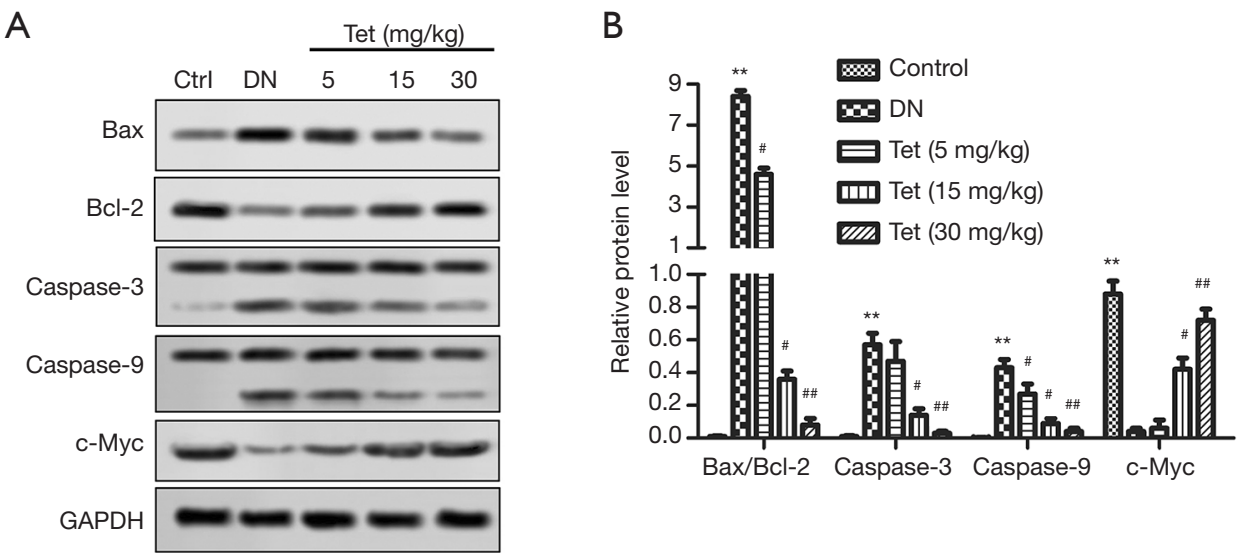

Figure 4 Tet relieved mitochondrial damage in the kidneys. Mitochondrial damage markers, Bax, Bcl-2, caspase-3, caspase-9 and c-Myc were detected by Western blot. (A) Representative pictures of Western blot. (B) Relative protein level of Bax/Bcl-2, caspase-3, caspase-9 and c-Myc. ${ }^{* *}, \mathrm{P}<0.01$ versus the control group; ${ }^{\#}, \mathrm{P}<0.05 ;{ }^{\# \#}, \mathrm{P}<0.01$ versus the $\mathrm{DN}$ model group). $\mathrm{DN}$, diabetic nephropathy; Tet, tetrandrine.

(Figure 6B,C), raised the levels of TNF- $\alpha$ and iNOS (Figure $6 D, E$ ), and increased the generation of ROS (Figure 6F). From Figure 6G, ML-385 also enhanced the relative protein levels of Bax/Bcl-2 and caspase-3. Generally, ML-385 seriously weakened the therapeutic effect of Tet on STZ-induced DN in rat model. The results suggested 
A
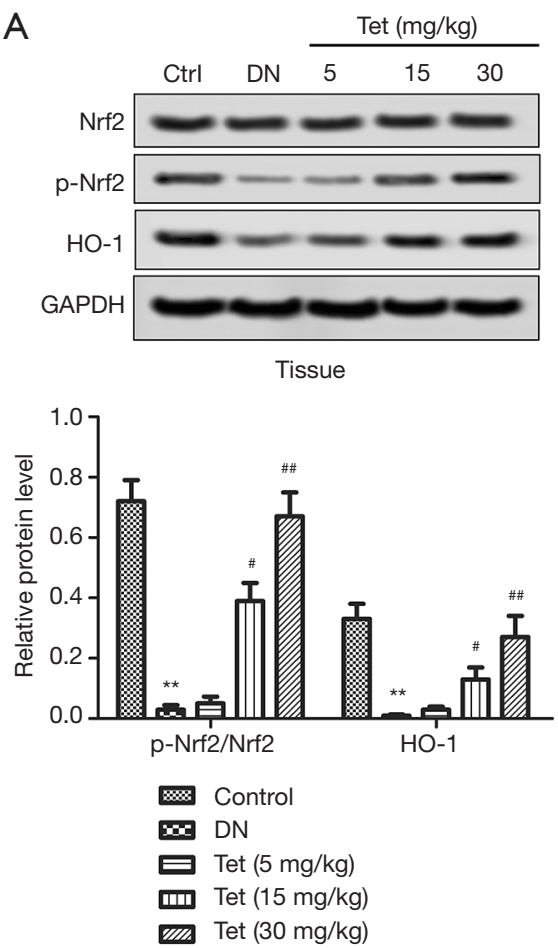

B
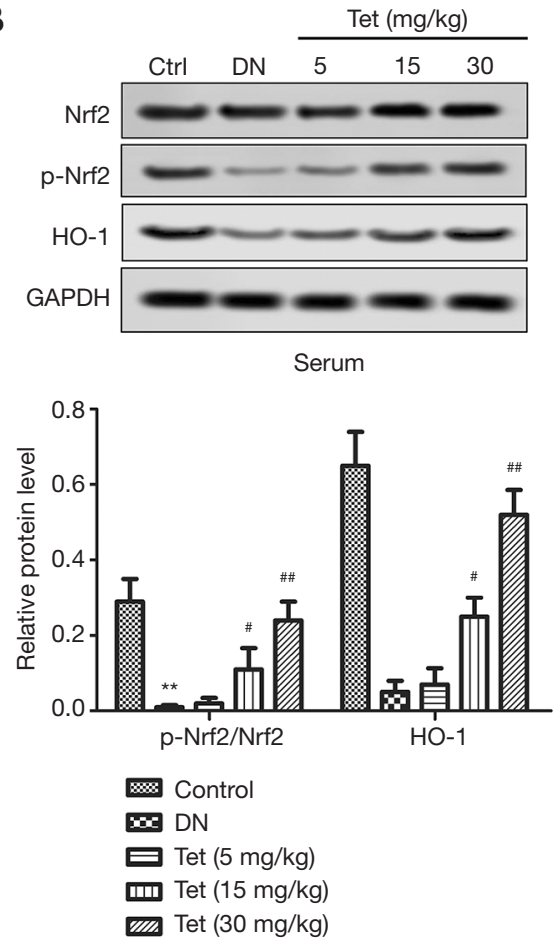

Figure 5 Tet activated Nrf2/HO-1 pathway in DN rat model. (A) Representative pictures of Western blot. Relative protein level of p-Nrf2/ Nrf2 and HO-1 in kidneys tissue. (B) Representative pictures of Western blot. Relative protein level of p-Nrf2/Nrf2 and HO-1 in serum. ${ }^{* *}$, $\mathrm{P}<0.01$ versus the control group; ${ }^{\#}, \mathrm{P}<0.05 ;{ }^{\# \#}, \mathrm{P}<0.01$ versus the $\mathrm{DN}$ model group). STZ, streptozotocin; DN, diabetic nephropathy; Nrf2, nuclear factor erythroid-derived 2-related factor 2; HO-1, heme oxygenase-1.

that the mediated role of Tet in STZ-induced DN was associated with $\mathrm{Nrf} 2 / \mathrm{HO}-1$ reactivation.

\section{Discussion}

Diabetes is one of the life-long severe diseases characterized by hyperglycemia caused by insulin secretion, insulin action, or both (2). Researchers had predicted that the incidence of diabetes would increase dramatically during the next two decades (22). Long-term hyperglycemia is related to microvascular and macrovascular complications and affects several organs, including eyes and kidneys. Thus, DN becomes one of the most common complications of diabetic patients (23). According to the statistics, about a quarter of diabetic patients develop kidney disease.

Of note, prompt treatment can prevent the deterioration of kidney disease. Hyperglycemia-mediated oxidative stress and inflammatory response are vital components in the pathogenesis of DN. Recent studies have shown that some antioxidants can protect renal function in a STZ- induced DN rat model (24). Tet is a type of effective antiinflammatory phytopharmaceuticals and has potential antioxidant (9) and anti-inflammatory activity (25); therefore, Tet can prevent the development of DN.

Blood glucose management is the primary method of controlling diabetes and its complications. According to reports by Al-Rasheed et al., diabetic rats exhibit hyperglycemia and weight loss in an STZ-induced diabetic rat model (26). The blood glucose level and body weight of diabetic rats treated with SIM were significantly improved (2). In this study, Tet decreased blood glucose, which showed that Tet is an effective treatment for DN. In the development of diabetes, nephropathies, including atrophic changes in the tubules, interstitium, and glomeruli, can be observed generally. This phenomenon leads to increased kidney weight, elevated serum CRE, and BUN (27). We found that hyperglycemia can cause renal injury, as shown by atrophic changes in the interstitium and elevated levels of CRE, urea, and BUN circulating. These findings were like the previous study in low-dose 
A
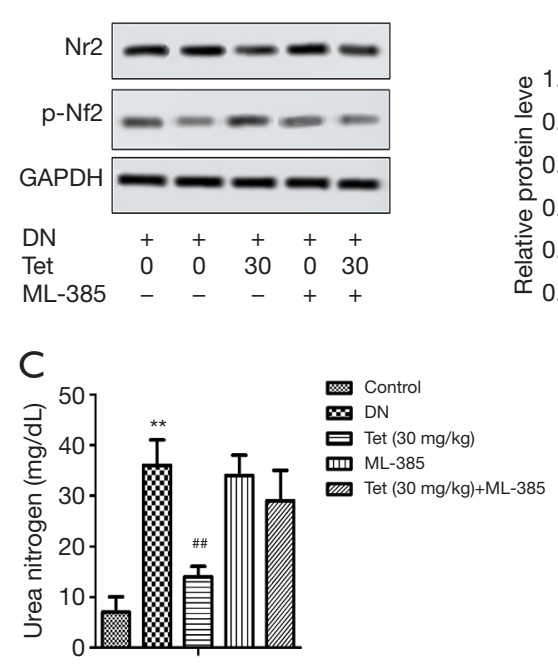

$\mathrm{F}$

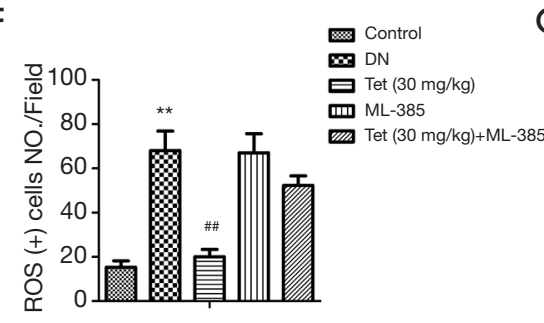

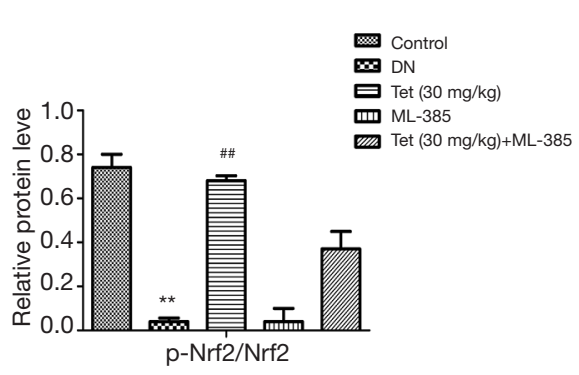

D

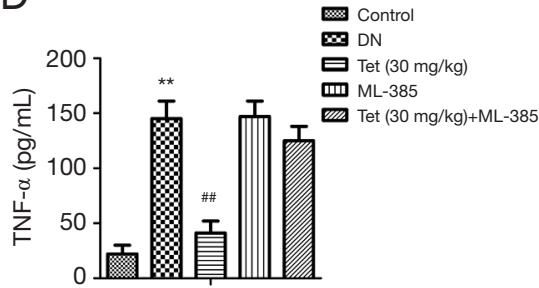

G

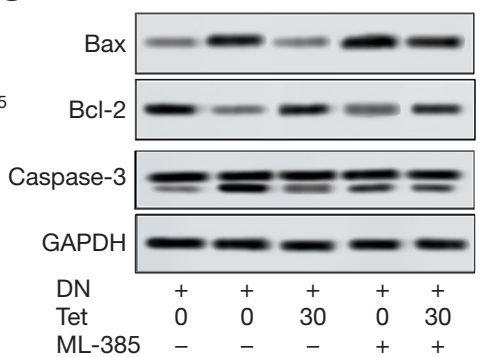

B

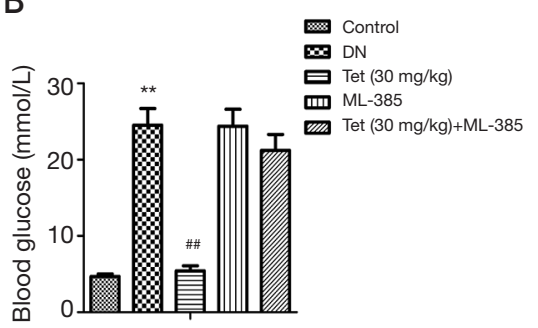

$\mathrm{E}$
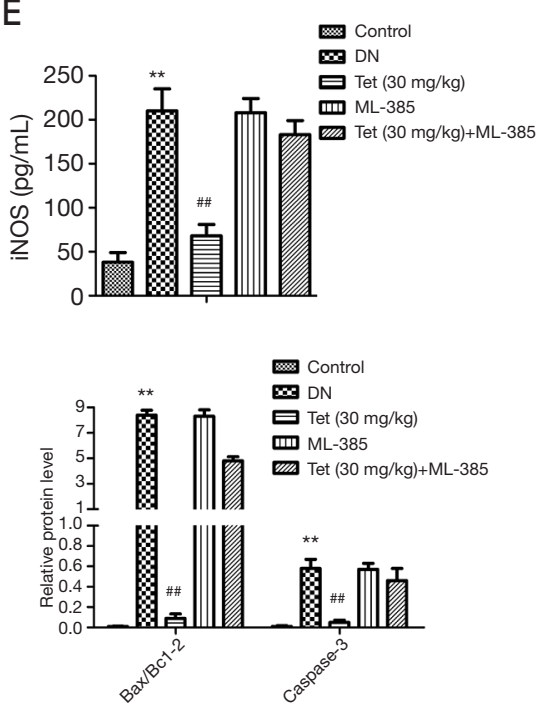

Figure 6 Tet mediated STZ-induced DN in rat model via Nrf2/HO-1 reactivation. (A) Representative pictures of Western blot. Relative protein level of p-Nrf2/Nrf2 in kidneys tissue; (B) the content of blood glucose; (C) the levels of urea nitrogen; (D) the levels of TNF- $\alpha$ (D) and iNOS (E) in kidneys tissue; (F) the generation of ROS in kidneys tissue; (G) representative pictures of Western blot. Relative protein level of Bax/Bcl-2 and caspase- 3 in kidneys tissue. ( ${ }^{* *}, \mathrm{P}<0.01$ versus the control group; ${ }^{\#}, \mathrm{P}<0.05 ;{ }^{\# \#,} \mathrm{P}<0.01$ versus the DN model group).

STZ-induced diabetic rats (28). Also, proteinuria levels increase. Proteinuria is a preliminary predictor of $\mathrm{DN}$ renal damage, and 24-hour urinary albumin excretion rate was detected to evaluate renal damage in patients with DN (29). Increased albumin excretion is due to podocyte hypertrophy and loss of foot process (30). Therefore, proteinuria can be improved by the reduction of kidney damage and glomerular hyperpermeability. Interestingly, Tet prevented kidney damage and the elevation of serum CRE, proteinuria, and urea nitrogen in STZ-induced diabetic rats.

Interleukin-10 (IL-10), interleukin-6 (IL-6), and tumor necrosis factor- $\alpha(\mathrm{TNF}-\alpha)$ are several strong inflammatory mediators that play essential roles in the occurrence and development of inflammation. These inflammatory mediators play pivotal roles in various cells, tissues, and organs. Following the effect of the inflammatory factors, the inflammatory mediator interleukin, interferon, $\mathrm{TNF}-\alpha$ were released from the body (31). Tet has the role of calcium antagonism, which showed by inhibiting calmodulin activity and reducing neutrophil calcium. Thus, we account that the inhibition of Tet on the release of TNF, IL-1, and PAF may be associated with its calcium antagonism. In the present study, the levels of inflammatory factors TNF- $\alpha$ and IL-6 were upregulated in DN and improved by Tet treatment. Conversely, the level of IL-10 was downregulated in DN and improved by Tet treatment. The downregulation might be another important mechanism of Tet anti-inflammatory and immunosuppressive effects.

iNOS is one of the three key enzymes that produce nitric oxide (NO) from amino acid 1-arginine. INOS-derived NO plays a vital role in many physiological, like blood pressure regulation, host defense mechanisms, and wound repair. To date, evidence has shown iNOS-derived NO play critical 
roles in the pathophysiological inflammation, infection, neoplastic diseases, liver cirrhosis, diabetes conditions (32). Liao et al. reported that the therapeutic effect of Huangqi injection on $\mathrm{DN}$ is related to the regulation of iNOS activity in macrophages (33). The result was consistent with the conclusion of the present study, the Tet's therapeutic effect on DN is related to the downregulation of iNOS level.

Oxidative stress and inflammatory response are inseparable because each reaction will produce and amplify another. Redox homeostasis and redox signaling are pivotal components of maintenance for a normal physiological steady-state (34). This study found that the lipid peroxidation product MDA increased significantly, LDH increased, and SOD antioxidant activity decreased in diabetic rats, which indicated there was an oxidation effect. Tet treatment significantly improved the adverse effects of this condition. Researchers had certified that Nrf2 and downstream antioxidants were upgraded in DN patient's kidneys (35). These researchers suggested that $\mathrm{Nrf} 2$ activation in $\mathrm{DN}$ initial stages is vital to prevent DN. Previous researches suggested that Tet is an effective activator of Nrf2 nuclear translocation in HepG2 cells (36). In the development process of diabetes, Nrf2 nuclear expression, and HO-1 levels were enhanced markedly. Researches had reported that the ratio of p-Nrf2/ $\mathrm{Nrf2}$ is therapeutic targets of DN (37). We found p-Nrf2/ $\mathrm{Nrf} 2$ was enhanced significantly in the diabetes group with Tet treatment, while addition of ML-385 weakened the role of Tet, which suggested Tet activated the Nrf2 pathway and elevated antioxidant activity. Therefore, we presumed that Tet can inhibit inflammation, oxidative stress and tissues apoptosis via $\mathrm{Nrf2}$ pathway, thereby, alleviate STZ-induced DN.

In conclusion, the present study implied that Tet is a potential therapeutic agent and can significantly restrain the diabetic process and renal damage in the treatment of the STZ-induced DN rat model. The underlying mechanism is associated with the reactivation of $\mathrm{Nrf} 2 / \mathrm{HO}-$ 1. Nevertheless, our study was operated on animal models with limited sample and clinical significance. Further research is needed to confirm Tet's protective effect on patients with $\mathrm{DN}$ damage.

\section{Acknowledgments}

The Shaanxi Traditional Chinese Medicine University Affiliated Hospital supported this study.
Funding: None.

\section{Footnote}

Reporting Checklist: The authors have completed the ARRIVE reporting checklist. Available at http://dx.doi. org/10.21037/atm-20-5548

Data Sharing Statement: Available at http://dx.doi. org/10.21037/atm-20-5548

Conflicts of Interest: All authors have completed the ICMJE uniform disclosure form (available at http://dx.doi. org/10.21037/atm-20-5548). The authors have no conflicts of interest to declare.

Ethical Statement: The authors are accountable for all aspects of the work in ensuring that questions related to the accuracy or integrity of any part of the work are appropriately investigated and resolved. The study was approved by the ethical committee of Shaanxi Traditional Chinese Medicine University Affiliated Hospital (Xianyang, China) (No. SXZY-1907). All animal experiments were conducted according to the ethical guidelines of Shaanxi Traditional Chinese Medicine University Affiliated Hospital (Xianyang, China).

Open Access Statement: This is an Open Access article distributed in accordance with the Creative Commons Attribution-NonCommercial-NoDerivs 4.0 International License (CC BY-NC-ND 4.0), which permits the noncommercial replication and distribution of the article with the strict proviso that no changes or edits are made and the original work is properly cited (including links to both the formal publication through the relevant DOI and the license). See: https://creativecommons.org/licenses/by-nc-nd/4.0/.

\section{References}

1. Soleymanian T, Kokabeh Z, Ramaghi R, et al. Clinical outcomes and quality of life in hemodialysis diabetic patients versus non-diabetics. J Nephropathol 2017;6:81-9.

2. Al-Rasheed NM, Al-Rasheed NM, Bassiouni YA, et al. Simvastatin ameliorates diabetic nephropathy by attenuating oxidative stress and apoptosis in a rat model of streptozotocin-induced type 1 diabetes. Biomed Pharmacother 2018;105:290-8.

3. Wagener FA, Dekker D, Berden JH, et al. The role of 
reactive oxygen species in apoptosis of the diabetic kidney. Apoptosis 2009;14:1451-8.

4. KDOQI Clinical Practice Guideline for Diabetes and CKD: 2012 Update. Am J Kidney Dis 2012;60:850-86.

5. Liu T, Liu X, Li W. Tetrandrine, a Chinese plant-derived alkaloid, is a potential candidate for cancer chemotherapy. Oncotarget 2016;7:40800-15.

6. Yuan X, Tong B, Dou Y, et al. Tetrandrine ameliorates collagen-induced arthritis in mice by restoring the balance between Th17 and Treg cells via the aryl hydrocarbon receptor. Biochem Pharmacol 2016;101:87-99.

7. Zhang J, Yu B, Zhang XQ, et al. Tetrandrine, an antihypertensive alkaloid, improves the sleep state of spontaneously hypertensive rats (SHRs). J Ethnopharmacol 2014;151:729-32.

8. Zou H, He T, Chen X. Tetrandrine inhibits differentiation of proinflammatory subsets of T helper cells but spares de novo differentiation of iTreg cells. Int Immunopharmacol 2019;69:307-12.

9. Song C, Ji Y, Zou G, et al. Tetrandrine down-regulates expression of miRNA-155 to inhibit signal-induced NF$\kappa \mathrm{B}$ activation in a rat model of diabetes mellitus. Int $\mathrm{J}$ Clin Exp Med 2015;8:4024-30.

10. Kang OH, An HJ, Kim SB, et al. Tetrandrine suppresses pro-inflammatory mediators in PMA plus A23187-induced HMC-1 cells. Int J Mol Med 2014;33:1335-40.

11. Bhagya N, Chandrashekar KR, Prabhu A, et al. Tetrandrine isolated from Cyclea peltata induces cytotoxicity and apoptosis through ROS and caspase pathways in breast and pancreatic cancer cells. In Vitro Cell Dev Biol Anim 2019;55:331-40.

12. Kobayashi M, Yamamoto M. Molecular mechanisms activating the Nrf2-Keap1 pathway of antioxidant gene regulation. Antioxid Redox Signal 2005;7:385-94.

13. Shelton LM, Park BK, Copple IM. Role of Nrf2 in protection against acute kidney injury. Kidney Int 2013;84:1090-5.

14. Copple IM. The Keap1-Nrf2 cell defense pathway-a promising therapeutic target? Adv Pharmacol 2012;63:43-79.

15. Zoja C, Benigni A, Remuzzi G. The Nrf2 pathway in the progression of renal disease. Nephrol Dial Transplant 2014;29 Suppl 1:i19-i24.

16. Zheng H, Whitman SA, Wu W, et al. Therapeutic potential of $\mathrm{Nrf} 2$ activators in streptozotocin-induced diabetic nephropathy. Diabetes 2011;60:3055-66.

17. Chen X, Wei SY, Li JS, Zhang QF, Wang YX, Zhao SL, et al. Overexpression of Heme Oxygenase-1
Prevents Renal Interstitial Inflammation and Fibrosis Induced by Unilateral Ureter Obstruction. PloS one. 2016;11:e0147084.

18. Kitada M, Ogura Y, Koya D. Rodent models of diabetic nephropathy: their utility and limitations. Int J Nephrol Renovasc Dis 2016;9:279-90.

19. Bao L, Li J, Zha D, et al. Chlorogenic acid prevents diabetic nephropathy by inhibiting oxidative stress and inflammation through modulation of the $\mathrm{Nrf} 2 / \mathrm{HO}-1$ and NF-кB pathways. Int Immunopharmacol 2018;54:245-53.

20. Hsu JH, Wu YC, Liou SS, et al. Mediation of Endogenous beta-endorphin by Tetrandrine to Lower Plasma Glucose in Streptozotocin-induced Diabetic Rats. Evid Based Complement Alternat Med 2004;1:193-201.

21. Lee NK, Choi YG, Baik JY, et al. A crucial role for reactive oxygen species in RANKL-induced osteoclast differentiation. Blood 2005;106:852-9.

22. Soler MJ, Batlle D. Single-cell RNA profiling of glomerular cells in diabetic kidney: a step forward for understanding diabetic nephropathy. Ann Transl Med 2019;7:S340.

23. Reutens AT, Atkins RC. Epidemiology of diabetic nephropathy. Contrib Nephrol 2011;170:1-7.

24. Hernández-Salinas R, Vielma AZ, Arismendi MN, et al. Boldine prevents renal alterations in diabetic rats. J Diabetes Res 2013;2013:593672.

25. Gao LN, Feng QS, Zhang XF, et al. Tetrandrine suppresses articular inflammatory response by inhibiting pro-inflammatory factors via NF- $\kappa \mathrm{B}$ inactivation. J Orthop Res 2016;34:1557-68.

26. Al-Rasheed NM, Al-Rasheed NM, Hasan IH, et al. Simvastatin Ameliorates Diabetic Cardiomyopathy by Attenuating Oxidative Stress and Inflammation in Rats. Oxid Med Cell Longev 2017;2017:1092015.

27. Cohen MP, Clements RS, Cohen JA, et al. Prevention of decline in renal function in the diabetic $\mathrm{db} / \mathrm{db}$ mouse. Diabetologia 1996;39:270-4.

28. Zhang S, Xu H, Yu X, et al. Metformin ameliorates diabetic nephropathy in a rat model of low-dose streptozotocin-induced diabetes. Exp Ther Med 2017;14:383-90.

29. Viberti G, Wheeldon NM. Microalbuminuria reduction with valsartan in patients with type 2 diabetes mellitus: a blood pressure-independent effect. Circulation 2002;106:672-8.

30. Reidy K, Kang HM, Hostetter T, et al. Molecular mechanisms of diabetic kidney disease. J Clin Invest 2014;124:2333-40. 


\section{Page 12 of 12}

31. Wu W, Jin $\mathrm{Y}$, Teng L, et al. Mitochondria-related reversal of early-stage diabetic nephropathy in donor kidney after transplantation in mice. Ann Transl Med 2019;7:801.

32. Lechner M, Lirk P, Rieder J. Inducible nitric oxide synthase (iNOS) in tumor biology: the two sides of the same coin. Semin Cancer Biol 2005;15:277-89.

33. Liao H, Hu L, Cheng X, et al. Are the Therapeutic Effects of Huangqi (Astragalus membranaceus) on Diabetic Nephropathy Correlated with Its Regulation of Macrophage iNOS Activity? J Immunol Res 2017;2017:3780572.

34. Ursini F, Maiorino M, Forman HJ. Redox homeostasis: The Golden Mean of healthy living. Redox Biol

Cite this article as: $\mathrm{Su} \mathrm{L}$, Cao $\mathrm{P}$, Wang $\mathrm{H}$. Tetrandrine mediates renal function and redox homeostasis in a streptozotocin-induced diabetic nephropathy rat model through Nrf2/HO-1 reactivation. Ann Transl Med 2020;8(16):990. doi: 10.21037/atm-20-5548

\section{Su et al. Tetrandrine mediates diabetic nephropathy}

2016;8:205-15.

35. Jiang T, Huang Z, Lin Y, et al. The protective role of Nrf2 in streptozotocin-induced diabetic nephropathy. Diabetes 2010;59:850-60.

36. Wang L, Ci X, Lv H, et al. Isotetrandrine ameliorates tert-butyl hydroperoxide-induced oxidative stress through upregulation of heme oxygenase-1 expression. Exp Biol Med (Maywood) 2016;241:1568-76.

37. Landis RC, Quimby KR, Greenidge AR. M1/M2 Macrophages in Diabetic Nephropathy: Nrf2/HO-1 as Therapeutic Targets. Curr Pharm Des 2018;24:2241-9.

(English Language Editor: J. Chapnick) 